\title{
Allelic Variation of the D4 Dopamine Receptor Polymorphic Region in Two Dog Breeds, Golden Retriever and Shiba
}

\author{
Youko NIIMI, Miho INOUE-MURAYAMA*, Yuichi MURAYAMA ${ }^{1)}$, Shin'ichi ITO and Toshiroh IWASAKI ${ }^{2,3)}$ \\ Department of Biological Diversity and Resources, Faculty of Agriculture, Gifu University, Gifu 501-1193, ${ }^{1) N a t i o n a l ~ I n s t i t u t e ~ o f ~ A n i m a l ~}$ \\ Health, Tsukuba 305-0856, 2) Veterinary Medical Teaching Hospital, Faculty of Agriculture, Gifu University, Gifu 501-1193, and \\ ${ }^{3}$ Present address: Department of Veterinary Internal Medicine, Tokyo University of Agriculture and Technology, Fuchu 183-8509, \\ Japan
}

(Received 13 July 1999/Accepted 17 August 1999)

ABSTRACT. The D4 dopamine receptor (D4DR) polymorphic region, which is possibly related to the personality trait known as novelty seeking in humans, was examined in $34 \mathrm{dogs}$ from two breeds (Golden retriever and the Japanese indigenous breed, Shiba) by the polymerase chain reaction (PCR), and the DNA sequences of each allele were determined. The polymorphic region of the dog D4DR gene was composed of 39- and 12- base pair (bp) units, and four alleles (A-D) were identified based on the number and/or order of these units. Intra- and inter-breed allele variations were observed. The frequency of the short A allele was dominant (78.9\%) in the Golden retriever, while the long D allele was most common (46.7\%) in the Shiba. These findings suggested that the allele frequency varied significantly between different breeds, and that analysis of the polymorphism in D4DR might be of use for understanding the behavioral traits of dogs.-KEY wORDS: breed, canine, D4 dopamine receptor, polymorphism, repetitive sequence.

The dog (Canis familiaris) has the longest history among domestic animals and has a close association with humans. More than 400 breeds have so far been established through breeding for various purposes, e.g., family dogs, working dogs for watching, herding, and hunting, etc. Purebred dogs are significantly different from each other, not only in their morphology but also in their behavioral traits $[6,7]$. This suggests that some behavioral traits are under genetic control. Although considerable progress has been made in dog genetics $[1,3,11]$, the detailed genetic background of the behavioral traits remains to be characterized.

In the synapses, chemical transmitting substances are released from the nerve endings and the transmission of stimuli is mediated by such substances interacting with the receptors expressed on adjacent neurons. The receptors for the above neurotransmitters are distributed in specific areas of the brain and are expressed in neural cells with effects upon various neurological and psychological functions. Genetic polymorphism has been reported in several genes encoding the above cell surface receptors. The polymorphism in some of these proteins has been found to be related to human personality. For example, human D4DR includes 2-10 tandem repeats of 16 amino acids in the third cytoplasmic loop. Benjamin et al. [2] and Ebstein et al. [4] reported that tandem repeat polymorphism in the D4 dopamine receptor is related to the human personality trait of novelty seeking. Individuals with long repeats (6 or more) in the D4DR gene revealed higher scores in

* Correspondence to: Inoue-Murayama, M., Department of Biological Diversity and Resources, Faculty of Agriculture, Gifu University, Gifu 501-1193, Japan. personality tests for novelty seeking than did those with shorter repeats.

In recent years, companion animals have become very important within human society. In particular, dogs play an essential role as guide animals, and in some cases for animal assisted therapy. Thus, if the D4DR gene is polymorphic and modulates the behavioral traits of dogs, screening of the D4DR gene may be useful in selecting dogs by aptitude for the above purposes. In the present study, we focused on the polymorphism of the dog D4DR gene. Golden retrievers and Shibas were examined because they are relevant breeds in Japan, and their behavioral traits were quite different [6, $7,16]$. We report the allelic variations and DNA sequences of the dog D4DR gene as observed in the Golden retriever and the Shiba.

\section{MATERIALS AND METHODS}

Peripheral blood samples were obtained by venipuncture from the saphena of 34 individuals of two dog breeds (19 from Golden retrievers, 15 from Shibas). The samples were collected at the Veterinary Medical Teaching Hospital, Gifu University, and the International Pet Culture Association (IPC), Okazaki. Shiba is the common name of a Japanese indigenous small-sized dog breed. Although three varieties, the Shinshu-Shiba (Nagano Prefecture), Mino-Shiba (Gifu and Nagano Prefectures) and San'in-Shiba (Tottori and Shimane Prefectures), have been established [17], the particular source(s) of our Shiba samples were unknown.

Genomic DNA was extracted by the phenol-chloroform method or with a QIAamp Blood Kit (QIAGEN, U.S.A.). The D4DR exon III polymorphic region was amplified by the PCR [13]. One hundred ng of the DNA was used in 20 
$\mu l$ PCR solution containing $0.5 \mu \mathrm{M}$ of forward D4F (5'TTCTTCCTACCCTGCCCGCTCATG-3') and reverse D4R (5'-GACCACCACCGGCAGGACCCTCAT-3') primers, 250 $\mu \mathrm{M}$ of dATP, dCTP and dTTP, $125 \mu \mathrm{M}$ of dGTP, $125 \mu \mathrm{M}$ of 7-deaza-dGTP (Boehringer-Mannheim, Germany), 5\% dimethyl sulfoxide, $20 \mathrm{mM}$ Tris- $\mathrm{HCl}(\mathrm{pH} \mathrm{8.8),} 10 \mathrm{mM} \mathrm{KCl}$, $2 \mathrm{mM} \mathrm{MgSO}_{4}, 0.1 \%$ triton X-100, $2 \mu \mathrm{g} \mathrm{BSA}$, and $2 \mathrm{U}$ Pfu Turbo DNA Polymerase (STRATAGENE, U.S.A.). D4F and D4R primer sequences were synthesized based on the human D4DR 4-repeat allele and the sequences employed correspond to the nucleotide positions of 3020 to 3043 and 3452 to 3475 , respectively [13]. After initial incubation at $98^{\circ} \mathrm{C}$ for $2 \mathrm{~min}, \mathrm{PCR}$ amplification was performed for 35 cycles composed of denaturation at $98^{\circ} \mathrm{C}$ for $30 \mathrm{sec}$, annealing at $65^{\circ} \mathrm{C}$ for $1 \mathrm{~min}$, and extension at $74^{\circ} \mathrm{C}$ for 1 min. This was followed by a final extension at $74^{\circ} \mathrm{C}$ for 10 min. The PCR products were first separated by electrophoresis on $2.5 \%$ agarose gel. They were then extracted from the gel and directly sequenced by the dye termination method employing a 377 DNA Sequencer (Perkin-Elmer, U.S.A.). To avoid nonspecific amplification, dog specific reverse primer, D4dogR (5'-CCGCGGGGGC TCTGCAGGGTCG-3'), was synthesized on the basis of the common region of dog sequences. A combination of D4F and D4dogR primers was used for the subsequent PCR. To facilitate the identification of genotypes of the same length, D4dogBR (5'-TGGGCTGGGGGTGCCGTCC-3') was designed and employed in combination with the $\mathrm{D} 4 \mathrm{~F}$ primer. A homology search of the sequence of the PCR product was carried out with registered sequences in GENBANK using the BLAST software $[9,10]$.

\section{RESULTS}

Figure 1 illustrates the results of the PCR amplification of genomic DNA in dogs. Three different sizes were observed on electrophoresis. Sequence analysis of these PCR products revealed that the dog sequences were most closely related to the D4DR sequence of humans [19], rats [14] and mice [5] among the registered sequences in GENBANK. Table 1 lists the percentages of homology among the dog, human and rat sequences. The sequences corresponding to the 5' upstream and the 3' downstream of human D4DR tandem repeats were comparably conserved among the dog, human and rat indicating that the dog sequences amplified by the PCR are derived from the D4DR gene. The sequences corresponding to human tandem repeats showed a relatively lower homology among the three species.

A comparison of the nucleotide sequences between the human 5-repeat allele and the allele observed in the Golden retriever (which we named the A allele) is given in Fig. 2. Although the human polymorphic region involves tandem repeats with a 48-bp unit, such a repeat structure was not identified in the corresponding region of dogs. The repetitive region of the dog sequence started at $24 \mathrm{bp}$ upstream compared with that of the human sequence.

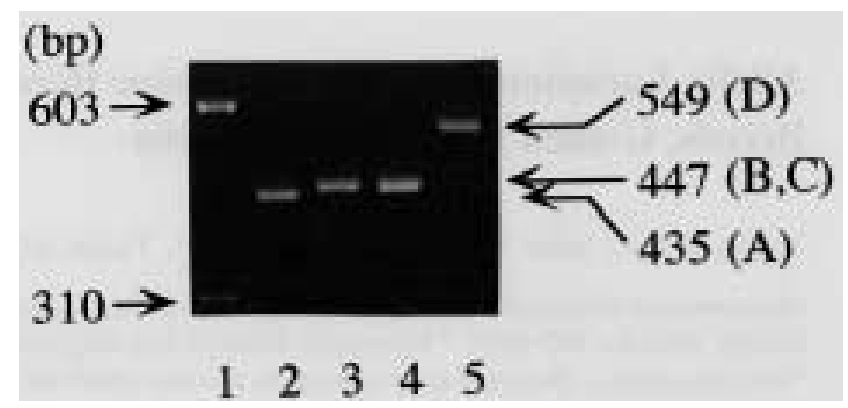

Fig. 1. Various alleles of the D4DR gene in dogs. A combination of D4F and D4dogR primers was used for the amplification. Lane 1: size marker, lane 2: A allele, lane 3: B allele, lane 4: C allele, and lane 5: D allele. The B and C alleles yielded PCR products with the same length, and could not be distinguished from each other without DNA sequencing. The product sizes were confirmed by sequence analysis.

Table 1. Sequence homology $y^{a)}$ of the D4DR gene among dog, human and rat

\begin{tabular}{cccc}
\hline & & Human & Rat \\
\hline 5' upstream & Dog & 87.0 & 82.8 \\
$(143 \mathrm{bp})^{\text {b) }}$ & Human & - & 86.3 \\
\hline 3' downstream & Dog & 79.1 & 67.3 \\
$(121 \mathrm{bp})^{\text {b) }}$ & Human & - & 72.5 \\
\hline & Dog & 55.9 & 49.5 \\
Repetitive region $^{\text {c }}$ & Human & - & 61.5 \\
\hline
\end{tabular}

a) Numbers indicate the percentages of homology. b) Numbers in parentheses indicate the length of the compared sequences. 5' upstream and 3' downstream sequences of dogs were identical in A, B, $\mathrm{C}$ and $\mathrm{D}$ alleles. c) The $\operatorname{dog} \mathrm{A}$ allele repetitive region and corresponding region of the rat were compared with the human 5-repeat (240 bp) and 2-repeat (96 bp) sequences, respectively.

Figure 3 shows the alignment of the repetitive region of 4 alleles found in dogs. The repetitive region of the dog sequence was composed of three types of units with different lengths of 39, 27, and $12 \mathrm{bp}$. The sequences of 27- and 12bp units were similar to those of the first and latter parts of the 39-bp unit, respectively. The number and order of these three units produced the observed allelic variation. The repetitive region of the shortest $\mathrm{A}$ allele was thus constructed with five 39-bp units, one 27-bp unit and one 12-bp unit. An additional 12-bp unit was inserted in the $\mathrm{B}$ and $\mathrm{C}$ alleles, although the insertion occurred at different positions. The longest D allele was constructed with seven 39-bp units, one 27-bp unit and four 12-bp units. Sequence comparisons revealed two unique substitutions in both the $\mathrm{A}$ and $\mathrm{B}$ alleles. In all alleles, no termination codon was found in the nucleotide sequences.

To identify the $\mathrm{B}$ and $\mathrm{C}$ alleles, which could not be distinguished by electrophoresis, the new reverse PCR primer, D4dogBR, was designed on the basis of nucleotide 

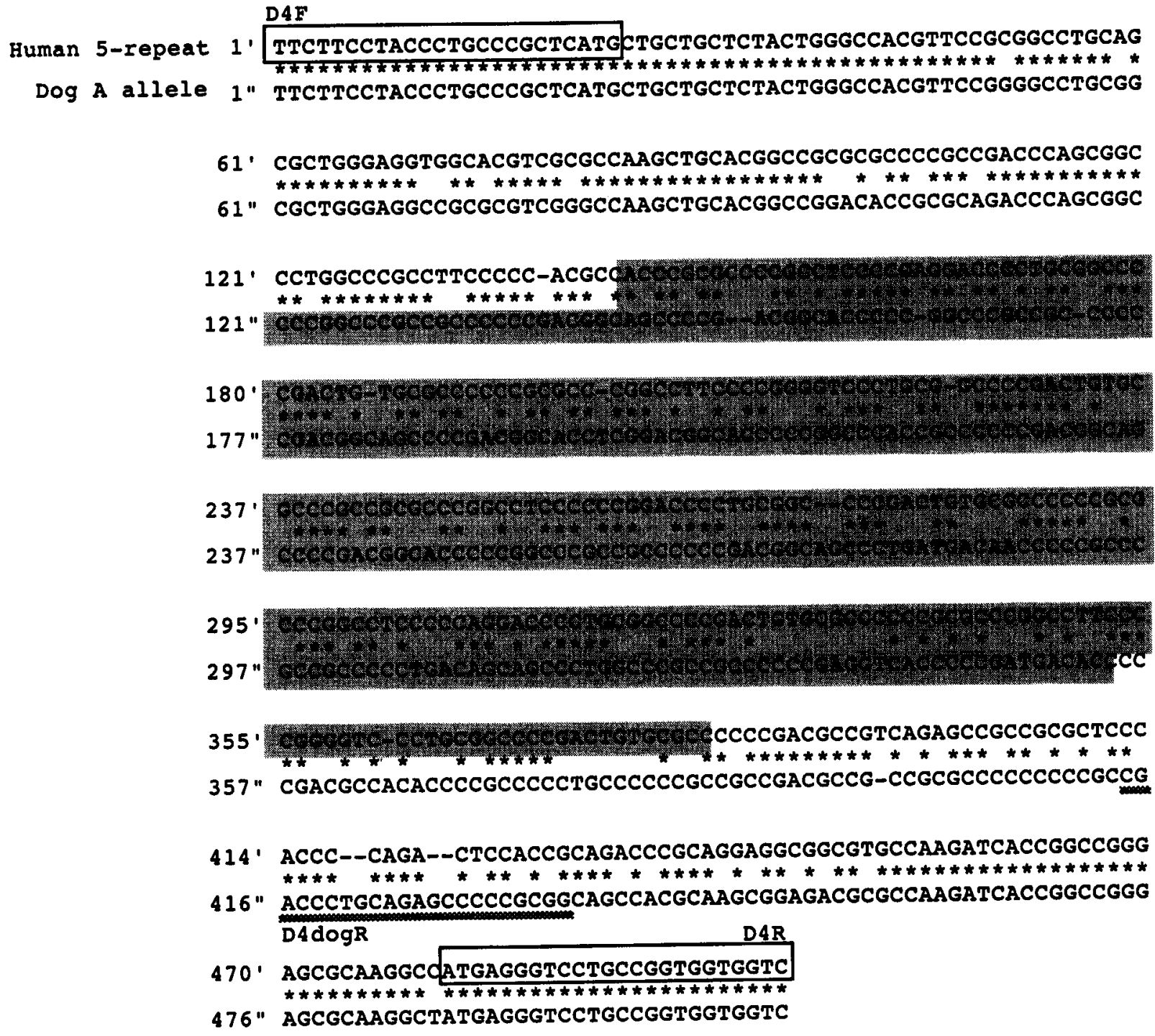

Fig. 2. Comparison of nucleotide sequences between the human D4DR 5-repeat allele and dog D4DR A allele. Asterisks and dashes indicate the same nucleotides and deletions, respectively. D4F and D4R primer sequences are enclosed in boxes. The newly designed reverse primer sequence for $\operatorname{dogs}(\mathrm{D} 4 \mathrm{dogR})$ is underlined. The repetitive regions for human and dog are shaded.

substitution at the 26th position in the first unit ( $G$ in allele $\mathrm{B}$ and $\mathrm{C}$ in allele $\mathrm{C})$. Figure 4 illustrates the identification of the $\mathrm{B}$ and $\mathrm{C}$ alleles by PCR amplification. The combination of D4F and D4dogBR primers amplified the PCR products with $168 \mathrm{bp}$ in $\mathrm{B}$ and $180 \mathrm{bp}$ in $\mathrm{C}$. This primer set was thus available to identify the $\mathrm{B}$ and $\mathrm{C}$ alleles.

The estimated genotypes of the individuals examined are listed in Table 2. In the Golden retriever, two alleles, A and $\mathrm{B}$, were observed and the $\mathrm{A}$ allele was the most common (78.9\%). On the other hand, the Shiba had three alleles (B, $\mathrm{C}$ and D), and the $\mathrm{C}(40.0 \%)$ and D alleles $(46.7 \%)$ were dominant. The observed heterozygosities in the Golden retriever and Shiba were $31.6 \%$ and $60.0 \%$, respectively. The actual number of alleles between the two breeds was significantly different based on a test of homogeneity $\left(\chi^{2}=57.198, \mathrm{df}=3, P<0.001\right)$.

\section{DISCUSSION}

Estimation of the repetitive region in the dog D4DR gene: The observed homology among the dog, human and rat sequences strongly suggested that the dog D4DR gene corresponding to the polymorphic region of the human D4DR gene could be amplified by the present PCR assay. In most primate species, polymorphism of the D4DR gene in terms of the repeat numbers of the 48-bp unit has been reported $[8,12]$. The repetitive region is absent in the mouse [5] and rat [14] sequences. The present study demonstrates that polymorphism of the D4DR gene exists in dogs. The polymorphic region was composed of 39- and 12-bp units, and the repeat structure of the dog D4DR gene was considerably different from those of primates. 


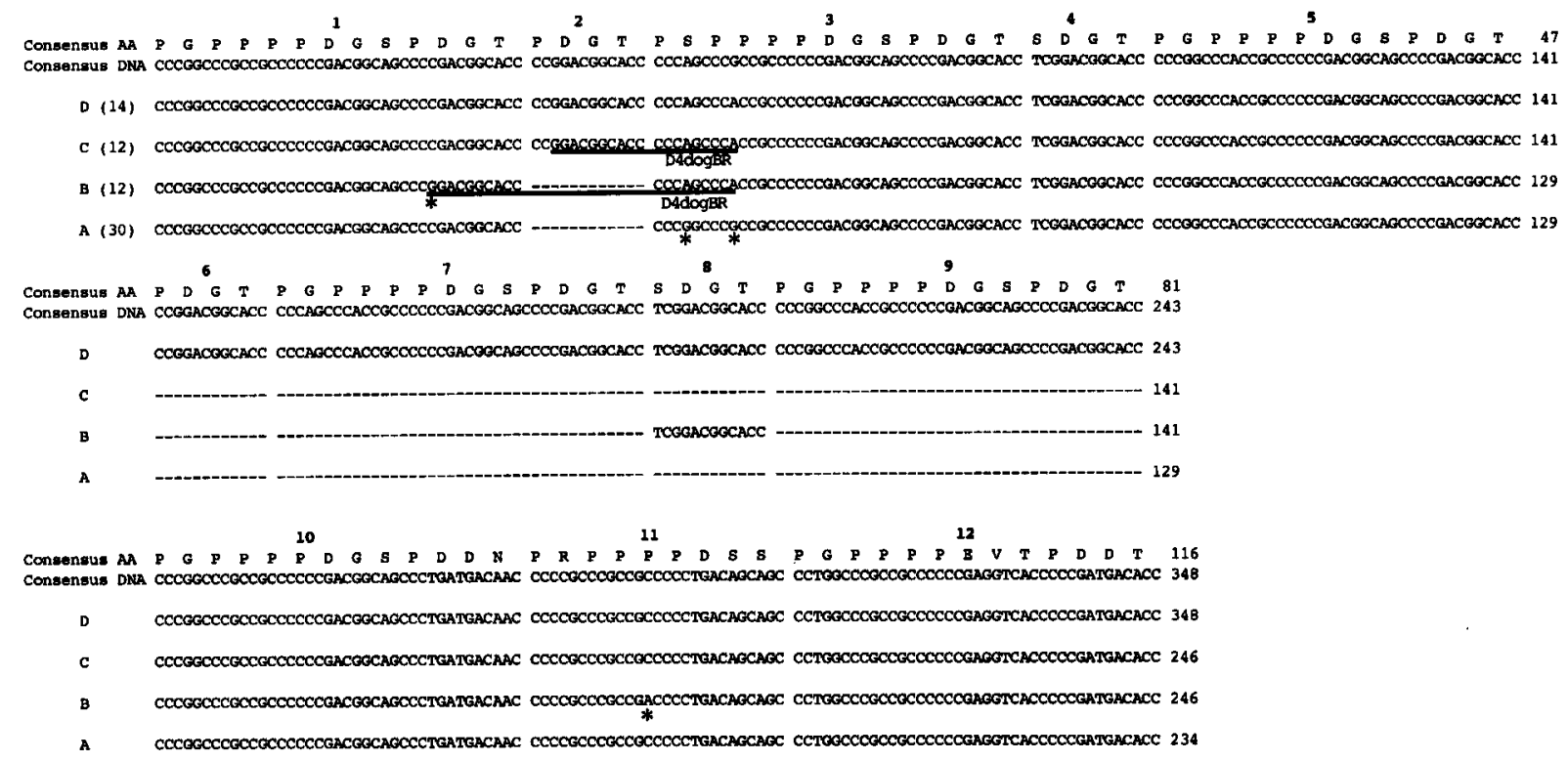

Fig. 3. Multiple alignment of D4DR gene repeat sequences from two dog breeds. The consensus nucleotide sequence and amino acid (AA) sequence are also given in the figure. The letters indicate the allele (A-D) of the sequence. The numbers in parentheses show the numbers of alleles observed in the dogs. Asterisks indicate nucleotide substitution. Amino acid substitutions occurred at the first (Ser to Gly) and the second (Pro to Thr) nucleotide substitution in the A and B alleles, respectively. The bold numbers the above sequences indicate the serial number of the units. The underlined sequences show the annealing sites of the reverse PCR primer, D4dogBR, employed for the identification of B and C. The sequences of A, B, C and D alleles can be obtained from the DDBJ/ EMBL/GenBank nucleotide sequence databases with the accession numbers, AB030234, AB030235, AB030236 and AB030237, respectively.

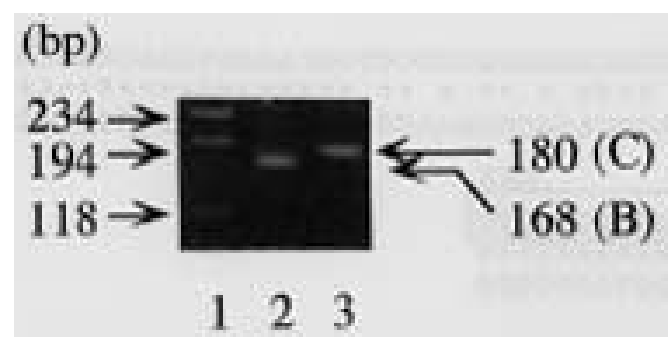

Fig. 4. Identification of the $\mathrm{B}$ and $\mathrm{C}$ alleles by PCR amplification. A combination of D4F primer and D4dogBR primer was used for the amplification. Lane 1: size marker, lane 2: B allele, and lane 3: C allele.

Evolutionary perspective of the repetitive region in the dog: In primates, the first and last units have relatively conserved sequences across species, indicating that various numbers of repeats might be produced by duplication of the internal repeat units [12]. Similarly, in dogs, the sequences of the first 39-bp unit and the last three units were the same except for two nucleotide substitutions in the $\mathrm{B}$ allele. However, a more complicated mechanism compared with primates appears to be included, since two units with different lengths (39 and $12 \mathrm{bp}$ ) were involved in the polymorphic region of the dog D4DR gene. The sequence from the 6th to 9th unit of the $\mathrm{D}$ allele is identical to those from the 2 nd to 5 th unit of the $\mathrm{C}$ and $\mathrm{D}$ alleles, and a duplication or deletion event of this sequence is therefore probably associated with the divergence of the $\mathrm{C}$ and $\mathrm{D}$ alleles.

The $\mathrm{C}$ and $\mathrm{D}$ alleles were dominant in the Shiba. The history of establishment of this breed started from the 1930's and is shorter than that of retrievers [15]. One Korean wolf (Canis lupus chanco) obtained from an individual kept at Kyoto Municipal Zoo had C/C genotype with the same nucleotide sequence as $\mathrm{C}$ allele of the Shiba (data not shown). These observations suggest that the Shiba may have a closer relation with the Korean wolf than Golden retriever. Although it has been estimated by comparing mitochondrial DNA sequences that wolves represented the ancestors of dogs [20], dog breeds could not be differentiated as a distinct cluster on the phylogenetic tree [18]. Additional samples from wolves are needed to make a precise estimation of the relationships among wolves and dog breeds based on D4DR alleles.

Allele A was observed only in the Golden retriever, whereas the B allele was shared by the Golden retriever and Shiba. All Golden retrievers except one had at least one A allele. This high frequency of the A allele in the Golden retriever may reflect the fact that this breed has been propagated from a small number of founders.

Relation between breed-specific characters and genetic differences: The allele frequencies of dog D4DR were significantly different in the Golden retriever and Shiba. The short A allele was dominant in the Golden retriever, 
Table 2. D4DR genotype in the dogs examined

\begin{tabular}{|c|c|c|c|}
\hline Breeds & $\begin{array}{l}\text { Specimen } \\
\text { number }\end{array}$ & \multicolumn{2}{|c|}{ Genotype } \\
\hline \multirow{19}{*}{ Golden retriever } & 1 & A & A \\
\hline & 2 & A & A \\
\hline & 3 & A & A \\
\hline & 4 & A & A \\
\hline & 5 & A & A \\
\hline & 6 & A & A \\
\hline & 7 & A & A \\
\hline & 8 & A & A \\
\hline & 9 & A & A \\
\hline & 10 & A & A \\
\hline & 11 & A & A \\
\hline & 12 & A & A \\
\hline & 13 & A & B \\
\hline & 14 & A & B \\
\hline & 15 & A & B \\
\hline & 16 & A & B \\
\hline & 17 & A & B \\
\hline & 18 & A & B \\
\hline & 19 & B & B \\
\hline \multirow[t]{15}{*}{ Shiba } & 1 & B & $\mathrm{C}$ \\
\hline & 2 & B & $\mathrm{C}$ \\
\hline & 3 & $\mathrm{~B}$ & $\mathrm{C}$ \\
\hline & 4 & B & $\mathrm{D}$ \\
\hline & 5 & $\mathrm{C}$ & $\mathrm{C}$ \\
\hline & 6 & $\mathrm{C}$ & $\mathrm{C}$ \\
\hline & 7 & $\mathrm{C}$ & $\mathrm{D}$ \\
\hline & 8 & $\mathrm{C}$ & $\mathrm{D}$ \\
\hline & 9 & $\mathrm{C}$ & $\mathrm{D}$ \\
\hline & 10 & $\mathrm{C}$ & $\mathrm{D}$ \\
\hline & 11 & $\mathrm{C}$ & $\mathrm{D}$ \\
\hline & 12 & $\mathrm{D}$ & $\mathrm{D}$ \\
\hline & 13 & $\mathrm{D}$ & $\mathrm{D}$ \\
\hline & 14 & $\mathrm{D}$ & $\mathrm{D}$ \\
\hline & 15 & $\mathrm{D}$ & $\mathrm{D}$ \\
\hline
\end{tabular}

while the long D allele was the most common in the Shiba. These two breeds are quite different in their behavioral traits. Hart et al. [6, 7] ranked 56 dog breeds on the basis of 13 behavioral traits: excitability, general activity, snapping at children, expressive barking, affection demand, territorial defense, watchdog barking, aggression to dogs, dominance over owner, obedience training, housebreaking ease, destructiveness, and playfulness. Tanabe et al. [16] added two behavioral traits: adaptability to new owners and friendliness to extra family members. The Golden retriever is shown to be suitable as a family dog by these criteria because of its high estimates for trainability and playfulness, and low estimates for aggression and territorial defense. On the other hand, Japanese indigenous breeds including the Shiba are suitable as watch dogs because of their low estimates for the former traits and high estimates for the latter traits. In humans, a significant relationship between the long allele (6 repeats or more) of the D4DR gene and a high score on personality tests for novelty seeking has been reported [2,4]. There is a possibility that allelic variation may be related to the above behavioral traits in the dog.
The Shiba has long C and D alleles at high frequency. D4DR is distributed mainly in the limbic system and is expressed in neural cells having an effect upon cognitive and emotional behaviors [19]. Therefore, allelic variation of D4DR gene may be related to dog behavioral traits such as excitability and aggression. However, the repeat structure of the D4DR polymorphic region differed between humans and dogs, and further research is clearly needed to elucidate the detailed relationships between D4DR polymorphism and the behavioral traits of dogs.

In Japan, most guide-dogs are Labrador retrievers or Golden retrievers. As a result of selection, these breeds might have acquired a high trainability, which is an invaluable characteristic for guide-dogs. The percentage of successfully passing the aptitude test for guide-dogs is about $30 \%$ among trained dogs, and there are insufficient guidedogs to satisfy the demand. Analysis of the genes affecting mental activity may be useful in selecting dogs for such practical use.

ACKNOWLEDGMENTS. We are indebted to Prof. Osamu Takenaka, Kyoto University, for the gift of the wolf DNA sample, and to the IPC for their cooperation in obtaining blood samples from dogs. We would like to thank Mr. B. B. Kayang, Gifu University, for reading our manuscript and making invaluable comments. This work was supported financially by the Gifu University Research Fund.

\section{REFERENCES}

1. Aguirre, G. D., Ray, K. and Acland, M. 1999. Overview of the international workshop on canine genetics. J. Hered. 90: $1-2$.

2. Benjamin, J., Li, L., Patterson, C., Greenberg, B. D., Murphy, D. L. and Hamer, D. H. 1996. Population and familial association between the $\mathrm{D} 4$ dopamine receptor gene and measures of novelty seeking. Nature Genet. 12: 81-84.

3. DogMap Consortium. 1999. DogMap: An international collaboration toward a low-resolution canine genetic marker map. J. Hered. 90: 3-6.

4. Ebstein, R. P., Novick, O., Umansky, R., Priel, B., Osher, Y., Blaine, D., Bennett, E. R., Nemanov, L., Katz, M. and Belmaker, R. H. 1996. Dopamine D4 receptor exon III polymorphism associated with the human personality trait of novelty seeking. Nature Genet. 12: 78-80.

5. Fishburn, C. S., Carmon, S. and Fuchs, S. 1995. Molecular cloning and characterization of the gene encoding the murine D4 dopamine receptor. FEBS Lett. 361: 215-219.

6. Hart, B. L. and Miller, M. F. 1985. Behavioral profiles of dog breeds. J. Am. Vet. Med. Assoc. 186: 1175-1180.

7. Hart, B. L. and Hart, L. A. 1985. Selecting pet dogs on the basis of cluster analysis of breed behavior profiles and gender. J. Am. Vet. Med. Assoc. 186: 1181-1185.

8. Inoue-Murayama, M., Takenaka, O. and Murayama, Y. 1998. Origin and divergence of tandem repeats of primate D4 dopamine receptor genes. Primates 39: 217-224.

9. Karlin, S. and Altschul, S. F . 1990. Methods for assessing the statistical significance of molecular sequence features by 
using general scoring schemes. Proc. Natl. Acad. Sci. U. S. A. 87: 2264-2268.

10. Karlin, S. and Altschul, S. F . 1993. Applications and statistics for multiple high-scoring segments in molecular sequences. Proc. Natl. Acad. Sci. U. S. A. 90: 5873-5877.

11. Langston, A. A., Mellersh, C. S., Wiegand, N. A., Acland, G. M., Ray, K., Aguirre, G. D. and Ostrander, E. A. 1999. Toward a framework linkage map of the canine genome. $J$. Hered. 90: 7-14.

12. Livak, K. J., Rogers, J. and Lichter, J. B. 1995. Variability of dopamine D4 receptor (DRD4) gene sequence within and among nonhuman primate species. Proc. Natl. Acad. Sci. U. S. A. 92: 427-431

13. Matsumoto, M., Hidaka, K., Tada, S., Tasaki, Y. and Yamaguchi, T. 1995. Polymorphic tandem repeats in dopamine D4receptor are spread over primate species. Biochem. Biophys. Res. Commn. 207: 467-475.

14. O'Malley, K. L., Harmon, S., Tang, L. and Todd, R. D. 1992. The rat dopamine D4 receptor: Sequence, gene structure, and demonstration of expression in the cardiovascular system. New Biol. 4: 137-146.

15. Ota, K. 1980. The dog, its domestication and development, with special reference to the origin of the Japanese native dog (a review). Rep. Soc. Res. Native Livestock 9: 53-94 and
232-235.

16. Tanabe, Y., Ogata, M., Kamiya, F. and Okabayashi, H. 1999. Breed difference in behavioral profiles of dogs: Quantitative analyses by veterinarians in Japan. Japan. J. Hum. Anim. Relat. 3: 92-98 (in Japanese).

17. Tanabe, Y., Ota, K., Ito, S., Hashimoto, Y., Sung. Y. Y., Ryu, J. K. and Faruque, M. O. 1991. Biochemical-genetic relationships among Asian and European dogs and the ancestry of the Japanese native dog. J. Anim. Breed Genet. 108: 455-478.

18. Tsuda, K., Kikkawa, Y., Yonekawa, H. and Tanabe, Y. 1997. Extensive interbreeding occurred among multiple matriarchal ancestors during the domestication of dogs: Evidence from inter- and intraspecies polymorphisms in the D-loop region of mitochondrial DNA between dogs and wolves. Genes Genet. Syst. 72: 229-238.

19. Van Tol, H. H. M., Bunzow, J. R., Guan, H. C., Sunahara, R. K., Seeman, P., Niznik, H. B. and Civelli, O. 1991. Cloning of the gene for a human dopamine D4 receptor with high affinity for the antipsychotic clozapine. Nature (Lond.) 350: 610-614.

20. Vila, C., Meldonado, J. E. and Wayne, R. K. 1999. Phylogenetic relationships, evolution, and genetic diversity of the domestic dog. J. Hered. 90: 71-77. 